University of Wollongong

Research Online

Faculty of Engineering and Information

Faculty of Engineering and Information

Sciences - Papers: Part B

Sciences

2018

The magneto-thermoelectric effect of graphene with intra-valley scattering

Wenye Duan

University of Wollongong,wduan@uow.edu.au

Junfeng Liu

South University of Science and Technology of China

C Zhang

University of Wollongong, czhang@uow.edu.au

Zhongshui Ma

Chinese Academy of Sciences, zma@uow.edu.au

Follow this and additional works at: https://ro.uow.edu.au/eispapers1

Part of the Engineering Commons, and the Science and Technology Studies Commons

Research Online is the open access institutional repository for the University of Wollongong. For further information contact the UOW Library: research-pubs@uow.edu.au 


\title{
The magneto-thermoelectric effect of graphene with intra-valley scattering
}

\author{
Abstract \\ We present a qualitative and quantitative study of the magneto-thermoelectric effect of graphene. In the \\ limit of impurity scattering length being much longer than the lattice constant, the intra-valley scattering \\ dominates the charge and thermal transport. The self-energy and the Green's functions are calculated in \\ the self-consistent Born approximation. It is found that the longitudinal thermal conductivity splits into \\ double peaks at high Landau levels and exhibits oscillations which are out of phase with the electric \\ conductivity. The chemical potential-dependent electrical resistivity, the thermal conductivities, the \\ Seebeck coefficient, and the Nernst coefficient are obtained. The results are in good agreement with the \\ experimental observations. \\ Disciplines \\ Engineering | Science and Technology Studies \\ Publication Details \\ Duan, W., Liu, J., Zhang, C. \& Ma, Z. (2018). The magneto-thermoelectric effect of graphene with intra- \\ valley scattering. Chinese Physics B, 27 (9), 097204-1-097204-8.
}

This journal article is available at Research Online: https://ro.uow.edu.au/eispapers1/2171 


\title{
The magneto-thermoelectric effect of graphene with intra-valley
}

\section{scattering *}

\author{
Wenye Duan(段文晔) $)^{1,2} \dagger$, Junfeng Liu(刘军丰) ${ }^{3}$, Chao Zhang(张潮 $)^{2}$, \\ and Zhongshui $\mathrm{Ma}$ (马中水 $)^{1,4}$ \\ ${ }^{1}$ School of Physics, Peking University, Beijing 100871, China \\ ${ }^{2}$ School of Physics, University of Wollongong, New South Wales 2522, Australia \\ ${ }^{3}$ Department of Physics, South University of Science and Technology of China, \\ Shenzhen 518055, China \\ ${ }^{4}$ Collaborative Innovation Center of Quantum Matter, Beijing, 100871, China
}

January 10, 2019

\begin{abstract}
We present a qualitative and quantitative study of magneto-thermoelectric effect of graphene. In the limit of impurity scattering length being much longer than the lattice constant, the intra-valley scattering dominates the charge and thermal transport. The self-energy and the Green's functions are calculated in the self-consistent Born approximation. It is found that the longitudinal thermal conductivity splits into double peaks at high Landau levels and exhibits oscillations which are out of phase with the electric conductivity. The chemical potential dependent electrical resistivity, the thermal conductivities, the Seebeck coefficient, and the Nernst coefficient have been obtained. The results are in good agreement with the experimental observations.
\end{abstract}

Keywords: graphene, thermoelectric transport, thermal transport

PACS: $72.80 . \mathrm{Vp}, 72.15 . J f, 73.50 . \mathrm{Lw}$

\section{Introduction}

Graphene, a single-atomic-thick honeycomb-like allotrope of carbon, has attracted intense interest since it was discovered in $2004^{[1]}$. On the basis of its potential applications in electronic devices, the electromagnetic responses in the graphene have been investigated. Its peculiar band structure, described by the effective massless Dirac Hamiltonian at low-energy ${ }^{[2-5]}$, results in a large amount of novel features in the electrical transport under magnetic field ${ }^{[6-14]}$. It has been shown that graphene has many unusual thermoelectric and thermal

*Project supported by the National Natural Science Foundation of China (Grant No. 11274013,11774006), NBRP of China (2012CB921300), and the Australian Research Council Grant (DP160101474).

†Corresponding author. E-mail: duanwy@pku.edu.cn 
transport properties ${ }^{[15-38]}$. Because of the sensitivity to the ambipolar behavior of graphene, the measurement of thermoelectric transport properties is regarded as an effective tool to deal with the additional characteristics of graphene, which are difficult to obtain from the electric conducting measurements. The Seebeck and Nernst signal in the graphene have been measured in the quantum Hall $(\mathrm{QH})$ regime ${ }^{[15-21]}$, in which the Seebeck coefficient displays a large peak and the Nernst signal displays a large oscillation at each Landau level (LL). The measurement of the Seebeck coefficient exhibits an abnormal sign change around the zeroth LL in comparison with other LLs ${ }^{[15-17,19]}$. Later, the extensions of the intrinsic thermal properties have been made towards the investigations of the thermoelectric effect in bilayer graphene ${ }^{[39-41]}$. There have been several theories put forward to explain the Seebeck and Nernst effects of graphene in the QH regime ${ }^{[42-50]}$. Some of the observed thermoelectric properties are not yet fully understood.

In this work, we perform a systematic study on the thermoelectric effect and thermal transport in the magnetically quantized graphene. We consider the scattering range of impurity much smaller than the electron wavelength and much longer than the lattice constant. In this limit the inter-valley scattering is much weaker than the intra-valley scattering. By employing the self-consistent Born approximation (SCBA), the Green's function and the self-energy are determined. Based on the Green's functions, all other coefficients of electrical, thermoelectrical, and thermal transport are calculated within the linear response theory. Although the thermal transport properties may be influenced by the acoustic and the optical phonon through inelastic electron-phonon scattering at high temperature, the electric contribution of graphene dominates over the phonon contribution at low temperatures. The temperature in the experiments have been confirmed below 50K ${ }^{[51-53]}$. Therefore, we neglect the effect of phonon on the graphene and concentrate on the influence of the impurity scattering in this work. The calculated results are in good agreement with the experimental findings.

The article is organized as follows. In the next section, we present the Hamiltonian describing the electrons under a strong magnetic field. The intra-valley interaction potential for the impurity scattering is introduced. The Green's function with a self-energy correction from the intra-valley impurity scattering is calculated within the SCBA. In Sec. III, we present the general relations between the thermoelectric transport coefficients. In Sec. IV, we calculate the thermoelectric response coefficients. The results have been compared with the experimental measurements. A summary is given in Sec. V.

\section{Hamiltonian and Green function in SCBA}

\subsection{The graphene in presence of a magnetic field}

Utilizing the $\boldsymbol{k} \cdot \boldsymbol{p}$ method or with the effective-mass approximation ${ }^{[54-58]}$, the nearest-neighbor tightbinding model of graphene without the impurity scattering leads to an effective Hamiltonian

$$
\mathcal{H}_{0}=v_{\mathrm{F}}\left(\tau_{0} \otimes \sigma_{x} p_{x}+\tau_{z} \otimes \sigma_{y} p_{y}\right),
$$

where $v_{\mathrm{F}}$ is the Fermi velocity. In this expression, $\sigma_{x}, \sigma_{y}$, and $\sigma_{z}$ are the Pauli matrices describing the pseudo spins, $\tau_{x}, \tau_{y}$, and $\tau_{z}$ are the Pauli matrices describing the valleys $\boldsymbol{K}$ and $\boldsymbol{K}^{\prime}$ in the reciprocal space, $\sigma_{0}$ and $\tau_{0}$ are $2 \times 2$ identity matrices. In the presence of a uniform magnetic field in the direction perpendicular to the graphene sheet, $\boldsymbol{p}$ is replaced by the mechanical momentum $\boldsymbol{\pi}=\boldsymbol{p}+e \boldsymbol{A}$. In the Landau gauge, the vector 
potential takes the form $\boldsymbol{A}=(0, B x, 0)$ (we use the SI units and electron charge is $-e(e>0))$. We shall neglect the Zeeman energy since it is much smaller than the LL spacing. The wave function should be written in a basis

$$
\Psi=\left(\psi_{A \boldsymbol{K}}, \psi_{B \boldsymbol{K}}, \psi_{A \boldsymbol{K}^{\prime}},-\psi_{B \boldsymbol{K}^{\prime}}\right)^{\mathcal{T}},
$$

where $\mathcal{T}$ stands for the transpose, $\psi_{(A / B)\left(K / K^{\prime}\right)}$ are the components of the spinors, and $A$ and $B$ represent the two inequivalent carbon atoms in the honeycomb hexagon lattices of graphene. The states can be obtained by solving the eigen equation $\mathcal{H}_{0} \Psi=\epsilon \Psi$. The eigenvalues are

$$
\epsilon_{n}=\operatorname{sgn}(n) \hbar \omega \sqrt{|n|}
$$

and the eigenstates are given by $\Psi_{n, k_{y}}^{\eta}(x, y)=C_{n}\left(1 / \sqrt{L_{y}}\right) \mathrm{e}^{\mathrm{i} k_{y} y} \Phi_{n, k_{y}}^{\eta}(x)$, with

$$
\Phi_{n, k_{y}}^{+}(x)=\left(-\mathrm{i} * \operatorname{sgn}(n) \phi_{|n|-1, k_{y}}(x), \phi_{|n|, k_{y}}(x), 0,0\right)^{\mathcal{T}},
$$

and

$$
\Phi_{n, k_{y}}^{-}(x)=\left(0,0, \phi_{|n|, k_{y}}(x),-\mathrm{i} * \operatorname{sgn}(n) \phi_{|n|-1, k_{y}}(x)\right)^{\mathcal{T}},
$$

where $C_{n}=1$ for $n=0$ and $1 / \sqrt{2}$ for $n \neq 0, \operatorname{sgn}(x)$ is the sign function,

$$
\phi_{|n|, k_{y}}(x)=\frac{\exp \left[-\frac{1}{2}\left(\frac{x+k_{y} l^{2}}{l}\right)^{2}\right]}{\left(2^{|n|}|n| ! \sqrt{\pi} l\right)^{1 / 2}} \mathrm{H}_{|n|}\left(\frac{x+k_{y} l^{2}}{l}\right),
$$

$n$ is a integer number, $\mathrm{H}_{n}(x)$ is the Hermite polynomial, $k_{y}$ is the wave vector along $y$ direction, $l=\sqrt{\hbar / e B}$, $\hbar \omega=\sqrt{2} \hbar v_{\mathrm{F}} / l$, and $\eta= \pm 1$ for the $\boldsymbol{K}(+)$ and $\boldsymbol{K}^{\prime}(-)$ valleys, respectively.

\subsection{Intra-valley impurity scattering}

Now we consider the impurity scattering. Utilizing the $\boldsymbol{k} \cdot \boldsymbol{p}$ method or with the effective-mass approximation ${ }^{[54-60]}$, the effective potential with impurity scatters at $\boldsymbol{r}_{i}^{\zeta}(\zeta=A, B)$ are given by

$$
U(\boldsymbol{r})=\sum_{\zeta, \boldsymbol{r}_{i}^{\zeta}}\left(\begin{array}{cccc}
u_{A}\left(\boldsymbol{r}-\boldsymbol{r}_{i}^{\zeta}\right) & 0 & u_{A}^{\prime}\left(\boldsymbol{r}-\boldsymbol{r}_{i}^{A}\right) \mathrm{e}^{\mathrm{i} \theta_{i}^{A}} & 0 \\
0 & u_{B}\left(\boldsymbol{r}-\boldsymbol{r}_{i}^{\zeta}\right) & 0 & u_{B}^{\prime}\left(\boldsymbol{r}-\boldsymbol{r}_{i}^{B}\right) \mathrm{e}^{\mathrm{i} \theta_{i}^{B}} \\
u_{A}^{\prime *}\left(\boldsymbol{r}-\boldsymbol{r}_{i}^{A}\right) \mathrm{e}^{-\mathrm{i} \theta_{i}^{A}} & 0 & u_{A}\left(\boldsymbol{r}-\boldsymbol{r}_{i}^{\zeta}\right) & 0 \\
0 & u_{B}^{\prime *}\left(\boldsymbol{r}-\boldsymbol{r}_{i}^{B}\right) \mathrm{e}^{-\mathrm{i} \theta_{i}^{B}} & 0 & u_{B}\left(\boldsymbol{r}-\boldsymbol{r}_{i}^{\zeta}\right)
\end{array}\right),
$$

where $\theta_{i}^{\zeta}=\left(\boldsymbol{K}^{\prime}-\boldsymbol{K}\right) \cdot \boldsymbol{r}_{i}^{\zeta}$. If the effective range of the impurity potential is much smaller than the typical electron wavelength, (which is the case near the Dirac point), the $\boldsymbol{r}$-dependent potentials can be approximated by $\delta\left(\boldsymbol{r}-\boldsymbol{r}_{i}\right)$. On the other hand, if the effective range of the impurity potential is larger than the lattice constant, the inter-valley scattering between $\boldsymbol{K}$ and $\boldsymbol{K}^{\prime}$ valleys can be neglected ${ }^{[56,58-60]}$. This argument was used to classify the intra-valley potentials as a type of long-range potentials by T. Ando et al. ${ }^{[56,60]}$. The scattering potential is written as,

$$
U_{i}(\boldsymbol{r})=\tau_{0} \otimes \sigma_{0} u_{i} \delta\left(\boldsymbol{r}-\boldsymbol{r}_{i}\right),
$$

where $\boldsymbol{r}_{i}$ is the impurity position and $u_{i}$ is the intensity of the scattering. The intra-valley scattering forbids the scattering between the states in $\boldsymbol{K}$ and $\boldsymbol{K}^{\prime}$ points. Including the scattering potential in Eq. (7), the full Hamiltonian is given by $\mathcal{H}=\mathcal{H}_{0}+U(\boldsymbol{r})$, where $U(\boldsymbol{r})=\sum_{i} U_{i}(\boldsymbol{r})$. 


\subsection{Green's function with the self-energy corrections in SCBA}

The Green's function is defined by $G(\epsilon)=[\epsilon-\mathcal{H}]^{-1}$. The matrix elements of unperturbed Green's function in the representation of the LL basis are diagonal, i.e., $G_{\alpha \alpha^{\prime}}^{(0)}(\epsilon)=\left\langle\alpha\left|\left[\epsilon-\mathcal{H}_{0}\right]^{-1}\right| \alpha^{\prime}\right\rangle=\delta_{\alpha \alpha^{\prime}} G_{\alpha}^{(0)}(\epsilon)$, where

$$
G_{\alpha}^{(0)}(\epsilon)=\frac{1}{\epsilon-\operatorname{sgn}(n) \hbar \omega \sqrt{|n|}},
$$

specified in a set of quantum numbers $\alpha=\left(\eta, n, k_{y}\right)$.

The electron-impurity scattering is studied through the impurity-averaged Green's function within the SCBA. The disorder term Eq. (7) is treated perturbatively with respect to the impurity-free Green's function $G_{\alpha}^{(0)}(\epsilon)$. Averaging over all possible configurations of random distributions of impurities, the matrix elements of $G(\epsilon)$ are given by Dyson's equation,

$$
\left\langle G_{\alpha \alpha^{\prime}}(\epsilon)\right\rangle=\delta_{\alpha \alpha^{\prime}} G_{\alpha}^{(0)}(\epsilon)+G_{\alpha}^{(0)}(\epsilon) \sum_{\alpha^{\prime}} \Sigma_{\alpha \alpha^{\prime}}(\epsilon)\left\langle G_{\alpha \alpha^{\prime}}(\epsilon)\right\rangle,
$$

where the self-energy $\Sigma_{\alpha \alpha^{\prime}}(\epsilon)$ is introduced within the SCBA,

$$
\Sigma_{\alpha \alpha^{\prime}}(\epsilon)=\sum_{\alpha_{1} \alpha_{1}^{\prime}}\left\langle U_{\alpha \alpha_{1}} U_{\alpha_{1}^{\prime} \alpha^{\prime}}(\epsilon)\right\rangle\left\langle G_{\alpha_{1} \alpha_{1}^{\prime}}(\epsilon)\right\rangle,
$$

and $G(\epsilon)=\left[\epsilon-\mathcal{H}_{0}-\Sigma(\epsilon)\right]^{-1}$. The average Green's function and the self-energy can be derived self-consistently for random impurities $\left\langle\left(u_{i}\right)^{2}\right\rangle=u^{2}$. The concentration of impurities is given by $n_{\mathrm{im}}$. The self-energy can be decomposed into a diagonal part $\bar{\Sigma}^{(\mathrm{D})}(\epsilon)$ and a off-diagonal part $\bar{\Sigma}^{(\mathrm{O})}(\epsilon)$, i.e.,

$$
\Sigma_{\alpha \alpha^{\prime}}(\epsilon)=\delta_{\alpha \alpha^{\prime}} \bar{\Sigma}^{(\mathrm{D})}(\epsilon)+\delta_{\alpha,-\alpha^{\prime}} \bar{\Sigma}^{(\mathrm{O})}(\epsilon),
$$

where $\pm \alpha=\left(\eta, \pm n, k_{y}\right)$ is a composite quantum number. The off-diagonal elements between the LL indices $+n$ and $-n$ are non vanishing. The self-energy terms in above equation can be written as

$$
\bar{\Sigma}^{(\mathrm{D})}(\epsilon)=\frac{V}{2}(\hbar \omega)^{2} \sum_{n=-N_{c}}^{N_{c}}\left[\bar{G}_{n}^{(\mathrm{D})}(\epsilon)+\bar{G}_{0}^{(\mathrm{O})}(\epsilon)\right],
$$

and

$$
\bar{\Sigma}^{(\mathrm{O})}(\epsilon)=\frac{V}{2}(\hbar \omega)^{2} \sum_{n=-N_{c}}^{N_{c}}\left[\bar{G}_{n}^{(\mathrm{O})}(\epsilon)+\bar{G}_{0}^{(\mathrm{D})}(\epsilon)\right],
$$

where the dimensionless parameter $V=n_{\mathrm{im}} u^{2} /\left(4 \pi v_{\mathrm{F}}^{2}\right)$ is a measure of the intensity of scattering within the SCBA.

The impurity-averaged Green's functions are reduced to the form

$$
\left\langle G_{\alpha \alpha^{\prime}}(\epsilon)\right\rangle=\delta_{\alpha \alpha^{\prime}} \bar{G}_{n}^{(\mathrm{D})}(\epsilon)+\delta_{\alpha,-\alpha^{\prime}} \bar{G}_{n}^{(\mathrm{O})}(\epsilon),
$$

where

$$
\bar{G}_{n}^{(\mathrm{D})}(\epsilon)=\frac{\epsilon+\epsilon_{n}-\bar{\Sigma}^{(\mathrm{D})}}{\left(\epsilon+\epsilon_{n}-\bar{\Sigma}^{(\mathrm{D})}\right)\left(\epsilon-\epsilon_{n}-\bar{\Sigma}^{(\mathrm{D})}\right)-\left(\bar{\Sigma}^{(\mathrm{O})}\right)^{2}},
$$

and

$$
\bar{G}_{n}^{(\mathrm{O})}(\epsilon)=\frac{\bar{\Sigma}^{(\mathrm{O})}}{\left(\epsilon+\epsilon_{n}-\bar{\Sigma}^{(\mathrm{D})}\right)\left(\epsilon-\epsilon_{n}-\bar{\Sigma}^{(\mathrm{D})}\right)-\left(\bar{\Sigma}^{(\mathrm{O})}\right)^{2}} .
$$

The scattering matrix elements between different quantum states are given by

$$
U_{n n^{\prime}, k_{y} k_{y}^{\prime}, \eta \eta^{\prime}}=u_{i} \delta_{q_{y}, k_{y}-k_{y}^{\prime}} \delta_{\eta \eta^{\prime}} \mathrm{e}^{-\mathrm{i} \boldsymbol{q} \cdot \boldsymbol{r}_{i}} C_{n} C_{n^{\prime}} \operatorname{sgn}(n) \operatorname{sgn}\left(n^{\prime}\right)\left[I_{|n|,\left|n^{\prime}\right|}+I_{|n|-1,\left|n^{\prime}\right|-1}\right]
$$


where

$$
I_{|n|,\left|n^{\prime}\right|}=(-1)^{\frac{M-N}{2}} \frac{N !\left(\frac{q_{x} l}{\sqrt{2}}\right)^{M-N} \mathrm{~L}_{N}^{(M-N)}\left(\left(\frac{q_{x} l}{\sqrt{2}}\right)^{2}\right)}{\sqrt{(|n|-1) ! \sqrt{\left(\left|n^{\prime}\right|-1\right) !}}} \exp \left[\frac{q_{x} l}{4}\left(\mathrm{i} 4 l k_{y}-q_{x} l\right)\right],
$$

$N=\min \left(|n|-1,\left|n^{\prime}\right|-1\right), M=\max \left(|n|-1,\left|n^{\prime}\right|-1\right)$, and $\mathrm{L}_{N}^{(M-N)}(x)$ is the associated Laguerre polynomial.

\section{Linear response theory and transport coefficients}

Within the linear response theory, under an applied field and a temperature gradient, the electrical current $\boldsymbol{j}^{C}$ and energy current $\boldsymbol{j}^{E}$ can be written in the general form:

$$
\boldsymbol{j}^{C}=\mathbb{L}^{C C} \cdot \boldsymbol{E}+\mathbb{L}^{C E} \cdot \boldsymbol{X}^{E}
$$

and

$$
\boldsymbol{j}^{E}=\mathbb{L}^{E C} \cdot \boldsymbol{E}+\mathbb{L}^{E E} \cdot \boldsymbol{X}^{E}
$$

where the external force for the temperature-gradient is expressed in the form $\boldsymbol{X}^{E}=T \nabla(1 / T)$. The coefficients $\mathbb{L}^{C C}, \mathbb{L}^{C E}, \mathbb{L}^{E C}$, and $\mathbb{L}^{E E}$ are general tensors. They can be expressed in terms of the current-current correlations $\mathbb{N}^{i j}$ together with the corrections from the magnetization $\mathbb{M}^{i j}, \mathbb{L}^{i j}=\mathbb{N}^{i j}+\mathbb{M}^{i j}[61-64]$, with the subscripts $i$ and $j$ (=C or $E$ ) standing for the charge and the energy currents in the current-current correlations. Utilizing the Kubo-Strěda formalism, the current-current correlation $\mathbb{N}^{i j}$ is given by

$$
\mathbb{N}_{a b}^{i j}(T, \mu)=\frac{\mathrm{i} \hbar}{L^{2}} \int_{-\infty}^{+\infty} \mathrm{d} \epsilon f_{\mathrm{F}}(\epsilon) \operatorname{Tr}\left\langle\left[J_{a}^{i} \frac{\mathrm{d} G\left(\epsilon_{+0}\right)}{\mathrm{d} \epsilon} J_{b}^{j} \delta(\epsilon-\mathcal{H})-J_{a}^{i} \delta(\epsilon-\mathcal{H}) J_{b}^{j} \frac{\mathrm{d} G\left(\epsilon_{-0}\right)}{\mathrm{d} \epsilon}\right]\right\rangle,
$$

while the corrections due to the magnetization $\mathbb{M}_{i j}$ are $\mathbb{M}_{C C}^{a b}=0$,

$$
\mathbb{M}_{a b}^{C E}=-\frac{e}{2 L^{2}} \int_{-\infty}^{+\infty} \mathrm{d} \epsilon f_{\mathrm{F}}(\epsilon) \operatorname{Tr}\left\langle\left[\delta(\epsilon-\mathcal{H})\left(r_{a} v_{b}-r_{b} v_{a}\right)\right]\right\rangle
$$

and

$$
\mathbb{M}_{a b}^{E E}=\frac{1}{L^{2}} \int_{-\infty}^{+\infty} \mathrm{d} \epsilon f_{\mathrm{F}}(\epsilon) \operatorname{Tr}\left\langle\left[\delta(\epsilon-\mathcal{H}) \mathcal{H}\left(r_{a} v_{b}-r_{b} v_{a}\right)\right]\right\rangle
$$

where $a$ and $b$ denote the spatial components $(x$ or $y), \epsilon_{ \pm 0}=\epsilon \pm \mathrm{i} 0, J_{a}^{C}\left(=-e v_{a}\right)$ and $J_{a}^{E}\left(=\left\{\mathcal{H}, v_{a}\right\} / 2\right)$ are the operators of charge current and energy flux, respectively, and $f_{\mathrm{F}}(\epsilon)=1 /\left(1+\mathrm{e}^{(\epsilon-\mu) / k_{\mathrm{B}} T}\right)$ is the equilibrium Fermi-Dirac distribution function, $L^{2}$ is the area of the system and the velocity operators $\boldsymbol{v}=(-\mathrm{i} / \hbar)[\boldsymbol{r}, \mathcal{H}]$. The physical meaning of the coefficients in Eqs. (18) and (19) are: $\mathbb{L}^{C C}=\sigma$ is the electrical conductivity, $(1 / T)\left[(\mu / e)+\mathbb{L}^{C C-1} \cdot \mathbb{L}^{C E}\right]=\boldsymbol{S}$ is the thermopower, $(1 / T)\left[\mathbb{L}^{E E}-\mathbb{L}^{E C} \cdot \mathbb{L}^{C C-1} \cdot \mathbb{L}^{C E}\right]=\boldsymbol{\kappa}$ is the thermal conductivity, and the thermoelectric figure of merit $Z T=\sigma_{x x} S_{x x}^{2} T / \kappa_{x x}$. The thermopower and the thermal conductivity are obtained under the condition of zero electric current.

The electrical conductivity is given by $\sigma_{a b}(T, \mu)=L_{a b}^{C C}(T, \mu)$ and can be written in the form

$$
\sigma_{a b}(T, \mu)=\sigma_{a b}^{I}(T, \mu)+\sigma_{a b}^{I I}(T, \mu) .
$$

$\sigma_{a b}^{I}(T, \mu)$ and $\sigma_{a b}^{I I}(T, \mu)$ can be obtained from the Green's functions by the following formula,

$$
\sigma_{a b}^{I}(T, \mu)=\int_{-\infty}^{+\infty} \mathrm{d} \epsilon\left[-\frac{\mathrm{d} f_{\mathrm{F}}(\epsilon)}{\mathrm{d} \epsilon}\right] \sigma_{a b}^{I}(0, \mu)
$$

and 


$$
\sigma_{a b}^{I I}(T, \mu)=\frac{g_{\mathrm{s}} e^{2} \hbar}{4 \pi L^{2}} \int_{-\infty}^{+\infty} \mathrm{d} \epsilon f_{\mathrm{F}}(\epsilon) \operatorname{Tr}\left\langle v_{a} \frac{\mathrm{d} G\left(\epsilon_{-0}\right)}{\mathrm{d} \epsilon} v_{b} G\left(\epsilon_{-0}\right)-v_{a} \frac{\mathrm{d} G\left(\epsilon_{+0}\right)}{\mathrm{d} \epsilon} v_{b} G\left(\epsilon_{+0}\right)-(a \leftrightarrow b)\right\rangle
$$

where

$$
\sigma_{a b}^{I}(0, \epsilon)=\frac{g_{\mathrm{s}} e^{2} \hbar}{4 \pi L^{2}} \operatorname{Tr}\left\langle v_{a}\left(G\left(\epsilon_{+0}\right)-G\left(\epsilon_{-0}\right)\right) v_{b} G\left(\epsilon_{-0}\right)-v_{a} G\left(\epsilon_{+0}\right) v_{b}\left(G\left(\epsilon_{+0}\right)-G\left(\epsilon_{-0}\right)\right)\right\rangle,
$$

$g_{\mathrm{s}}$ is the degeneracy of spin. Applying the relations $\boldsymbol{v}=(-\mathrm{i} / \hbar)[\boldsymbol{r}, \mathcal{H}]$ and $d G(\epsilon \pm \mathrm{i} 0) / d \epsilon=-[G(\epsilon \pm \mathrm{i} 0)]^{2}$, $\sigma_{a b}^{I I}(T, \mu)$ can be rewritten as

$$
\sigma_{a b}^{I I}(T, \mu)=\int_{-\infty}^{+\infty} \mathrm{d} \epsilon\left[-\frac{\mathrm{d} f_{\mathrm{F}}(\epsilon)}{\mathrm{d} \epsilon}\right] \sigma_{a b}^{I I}(0, \epsilon)
$$

where

$$
\sigma_{a b}^{I I}(0, \epsilon)=-\frac{g_{\mathrm{s}} e^{2}}{4 \mathrm{i} \pi L^{2}} \operatorname{Tr}\left\langle\left(r_{a} v_{b}-r_{b} v_{a}\right)\left(G\left(\epsilon_{+0}\right)-G\left(\epsilon_{-0}\right)\right)\right\rangle,
$$

The electrical conductivity is expressed as

$$
\sigma_{a b}(T, \mu)=\int \mathrm{d} \epsilon\left[-\frac{\mathrm{d} f_{\mathrm{F}}(\epsilon)}{\mathrm{d} \epsilon}\right] \sigma_{a b}(0, \epsilon)
$$

with $\sigma_{a b}(0, \epsilon)=\sigma_{a b}^{I}(0, \epsilon)+\sigma_{a b}^{I I}(0, \epsilon)$. In the calculations of $\sigma_{a b}(0, \mu)$, we need to use the matrix elements of the velocity operators in the representation of the LL basis

$$
\begin{gathered}
v_{x, \alpha \alpha^{\prime}}=v_{F} C_{n^{\prime}} C_{n} \delta_{\kappa \kappa^{\prime}} \delta_{k_{y} k_{y}^{\prime}}\left[\mathrm{i} * \operatorname{sgn}\left(n^{\prime}\right) \delta_{|n|+1,\left|n^{\prime}\right|}-\mathrm{i} * \operatorname{sgn}(n) \delta_{|n|-1,\left|n^{\prime}\right|}\right], \\
v_{y, \alpha \alpha^{\prime}}=v_{F} C_{n} C_{n^{\prime}} \delta_{\kappa \kappa^{\prime}} \delta_{k_{y} k_{y}^{\prime}}\left[\operatorname{sgn}(n) \delta_{|n|-1,\left|n^{\prime}\right|}+\operatorname{sgn}\left(n^{\prime}\right) \delta_{|n|+1,\left|n^{\prime}\right|}\right],
\end{gathered}
$$

and the vortex correction in the SCBA

$$
\Gamma=\left\langle G(\epsilon) v_{a} G(\epsilon)\right\rangle=\langle G(\epsilon)\rangle v_{a}\langle G(\epsilon)\rangle+\langle G(\epsilon)\rangle\langle U \Gamma U\rangle\langle G(\epsilon)\rangle
$$

Similar with the conductivity, it has been shown that $\mathbb{L}_{a b}^{C E}(T, \mu)$ and $\mathbb{L}_{a b}^{E E}(T, \mu)$ can be obtained in terms of the $\sigma_{a b}(0, \epsilon)$ through the following relations ${ }^{[61,63,65]}$

$$
\mathbb{L}_{a b}^{C E}(T, \mu)=\mathbb{L}_{a b}^{E C}(T, \mu)=-\frac{1}{e} \int \mathrm{d} \epsilon\left[-\frac{\mathrm{d} f_{F}(\epsilon)}{\mathrm{d} \epsilon}\right] \epsilon \sigma_{a b}(0, \epsilon),
$$

and

$$
\mathbb{L}_{a b}^{E E}(T, \mu)=\frac{1}{e^{2}} \int \mathrm{d} \epsilon\left[-\frac{\mathrm{d} f_{\mathrm{F}}(\epsilon)}{\mathrm{d} \epsilon}\right] \epsilon^{2} \sigma_{a b}(0, \epsilon)
$$

\section{The magneto-transport coefficients of graphene}

The thermoelectric and thermal response coefficients can be obtained by Eqs. (29), (33), and (34). In the following subsection, we will evaluate the conductivity tensor by the Green's functions within the SCBA. 


\subsection{Analytical formula for calculating the conductivity}

\subsubsection{The longitudinal conductivity}

The longitudinal conductivity is $\sigma_{x x}$. Since $\left(r_{x} v_{x}-r_{x} v_{x}\right)=0$, therefore Eq. (28) shows $\sigma_{x x}^{I I}(0, \epsilon)=0$. The longitudinal conductivity is $\sigma_{x x}(T, \mu)=\sigma_{x x}^{I}(T, \mu)=\int_{-\infty}^{+\infty} \mathrm{d} \epsilon\left[-\left(\mathrm{d} f_{F}(\epsilon) / \mathrm{d} \epsilon\right)\right] \sigma_{x x}^{I}(0, \epsilon)$, where

$$
\sigma_{x x}^{I}(0, \epsilon)=2 Y_{x x}\left(\epsilon_{+0}, \epsilon_{-0}\right)-Y_{x x}\left(\epsilon_{+0}, \epsilon_{+0}\right)-Y_{x x}\left(\epsilon_{-0}, \epsilon_{-0}\right),
$$

with

$$
Y_{a b}\left(\epsilon, \epsilon^{\prime}\right)=\frac{g_{\mathrm{s}} e^{2} \hbar}{4 \pi L^{2}} \operatorname{Tr}\left\langle v_{a} G(\epsilon) v_{b} G\left(\epsilon^{\prime}\right)\right\rangle .
$$

Taking account of the impurity scattering within SCBA, it is found that

$$
Y_{x x}\left(\epsilon, \epsilon^{\prime}\right)=\frac{g_{\mathrm{s}} e^{2}}{2 \pi h} \sum_{\beta=1,2} \frac{\varphi^{\beta}\left(\epsilon, \epsilon^{\prime}\right)}{\left(1-2 V \varphi^{\beta}\left(\epsilon, \epsilon^{\prime}\right)\right)},
$$

where $\varphi^{1}\left(\epsilon, \epsilon^{\prime}\right)=\left[(\hbar \omega)^{2} / 2\right] \sum_{n \geq 0} \tilde{g}_{n+1}^{-}(\epsilon) \tilde{g}_{n}^{+}\left(\epsilon^{\prime}\right)$ and $\varphi^{2}\left(\epsilon, \epsilon^{\prime}\right)=\left[(\hbar \omega)^{2} / 2\right] \sum_{n \geq 0} \tilde{g}_{n}^{+}(\epsilon) \tilde{g}_{n+1}^{-}\left(\epsilon^{\prime}\right)$. We define $\tilde{g}_{n}^{ \pm}(\epsilon)$ as $\tilde{g}_{n}^{ \pm}(\epsilon) \equiv(1 / 2)\left[\tilde{G}_{n}^{ \pm}(\epsilon)+\tilde{G}_{-n}^{ \pm}(\epsilon)\right]$, where $\tilde{G}_{n}^{ \pm}(\epsilon) \equiv \bar{G}_{n}^{(\mathrm{D})}(\epsilon) \pm \bar{G}_{n}^{(\mathrm{O})}(\epsilon)$. The self-energy can be obtained by self-consistently solving the equation

$$
\tilde{\Sigma}^{ \pm}(\epsilon)=V(\hbar \omega)^{2} \sum_{n=0}^{N_{c}} \frac{\epsilon-\tilde{\Sigma}^{\mp}}{\left(\epsilon-\tilde{\Sigma}^{+}\right)\left(\epsilon-\tilde{\Sigma}^{-}\right)-\epsilon_{n}^{2}} .
$$

Then the Green's functions $\tilde{G}_{n}^{ \pm}(\epsilon)$ are obtained $\tilde{G}_{n}^{ \pm}(\epsilon)=\left(\epsilon+\epsilon_{n}-\tilde{\Sigma}^{\mp}\right) /\left[\left(\epsilon-\tilde{\Sigma}^{+}\right)\left(\epsilon-\tilde{\Sigma}^{-}\right)-\epsilon_{n}^{2}\right]$, so that $\tilde{g}_{n}^{ \pm}(\epsilon)=\left(\epsilon-\tilde{\Sigma}^{\mp}\right) /\left[\left(\epsilon-\tilde{\Sigma}^{+}\right)\left(\epsilon-\tilde{\Sigma}^{-}\right)-\epsilon_{n}^{2}\right]$.

\subsubsection{The Hall conductivity}

We now calculate the Hall conductivity $\sigma_{x y} . \sigma_{x y}^{I}(0, \epsilon)$ is found as

$$
\sigma_{x y}^{I}(0, \epsilon)=2 Y_{x y}\left(\epsilon_{+0}, \epsilon_{-0}\right)-Y_{x y}\left(\epsilon_{+0}, \epsilon_{+0}\right)-Y_{x y}\left(\epsilon_{-0}, \epsilon_{-0}\right),
$$

where

$$
Y_{x y}\left(\epsilon, \epsilon^{\prime}\right)=\frac{g_{\mathrm{s}} e^{2}}{2 \pi h} \sum_{\beta=1,2}(-1)^{\beta+1} \frac{\varphi^{\beta}\left(\epsilon, \epsilon^{\prime}\right)}{\left(1-2 V \varphi^{\beta}\left(\epsilon, \epsilon^{\prime}\right)\right)} .
$$

We have $\sigma_{x y}^{I}(T, \mu)=\int_{-\infty}^{+\infty} \mathrm{d} \epsilon\left[-\left(\mathrm{d} f_{\mathrm{F}}(\epsilon) / d \epsilon\right)\right] \sigma_{x y}^{I}(0, \epsilon)$.

$\sigma_{x y}^{I I}(0, \epsilon)$ is found as

$$
\sigma_{x y}^{I I}(0, \epsilon)=\frac{g_{\mathrm{s}} e^{2}}{2 L^{2}} \operatorname{Tr}\left\langle\left(r_{x} v_{y}-r_{y} v_{x}\right) \delta(\epsilon-H)\right\rangle,
$$

where we have used the relation $\delta(\epsilon-H)=(\mathrm{i} / 2 \pi)\left[G\left(\epsilon_{+0}\right)-G\left(\epsilon_{-0}\right)\right] . \sigma_{x y}^{I I}(T, \mu)$ can be written as

$$
\sigma_{x y}^{I I}(T, \mu)=\frac{g_{\mathrm{s}} e^{2}}{2 L^{2}} \int_{-\infty}^{+\infty} \mathrm{d} \epsilon f_{\mathrm{F}}(\epsilon) \operatorname{Tr}\left\langle\left(r_{x} v_{y}-r_{y} v_{x}\right) \frac{\mathrm{d} \delta(\epsilon-H)}{\mathrm{d} \epsilon}\right\rangle .
$$

Under the Landau gauge, we have $\boldsymbol{A}=B(0, x, 0)$ for the magnetic field along the z-axis $\boldsymbol{B}=(0,0, B)$. The terms in the trace can be written in the form $(1 / 2) \operatorname{Tr}\left[\left(r_{x} v_{y}-r_{y} v_{x}\right) \mathrm{d} \delta(\epsilon-\mathcal{H}) / \mathrm{d} \epsilon\right]=-(1 / e) \partial[\operatorname{Tr} \delta(\epsilon-\mathcal{H})] / \partial B$. $\sigma_{x y}^{I I}(T, \mu)$ becomes $\sigma_{x y}^{I I}(T, \mu)=-\left(g_{\mathrm{s}} e / L^{2}\right) \int_{-\infty}^{+\infty} \mathrm{d} \epsilon f_{\mathrm{F}}(\epsilon)\langle\partial \operatorname{Tr} \delta(\epsilon-\mathcal{H}) / \partial B\rangle$. Because the particle number is found as $\mathcal{N}(T, \mu)=g_{\mathrm{s}} \int_{-\infty}^{+\infty} \mathrm{d} \epsilon f_{\mathrm{F}}(\epsilon) \operatorname{Tr} \delta(\epsilon-\mathcal{H}), \sigma_{x y}^{I I}(T, \mu)$ is expressed in terms of the particle number 
$\sigma_{x y}^{I I}(T, \mu)=-\left(e / L^{2}\right)\left\langle(\partial \mathcal{N}(T, \mu) / \partial B)_{\mu}\right\rangle$. Utilizing the density of state $D(\epsilon)=-\left(g_{\mathrm{s}} / \pi^{2} l^{2}\right) \sum_{n} \operatorname{Im}\left\langle G_{n n}\left(\epsilon_{+0}\right)\right\rangle$ and $\mathcal{N}(T, \mu)=-\left(g_{\mathrm{s}} e B / \pi^{2} \hbar\right) \int_{-\infty}^{+\infty} \mathrm{d} \epsilon f_{\mathrm{F}}(\epsilon) \sum_{n} \operatorname{Im}\left\langle G_{n n}\left(\epsilon_{+0}\right)\right\rangle, \sigma_{x y}^{I I}(T, \mu)$ is found as

$$
\sigma_{x y}^{I I}(T, \mu)=\frac{2 g_{\mathrm{s}} e^{2}}{\pi h} \int_{-\infty}^{+\infty} \mathrm{d} \epsilon f_{\mathrm{F}}(\epsilon) \sum_{n} \operatorname{Im}\left\langle G_{n n}\left(\epsilon_{+0}\right)\right\rangle+\frac{g_{\mathrm{s}} e^{2}}{\pi h} \int_{-\infty}^{+\infty} \mathrm{d} \epsilon \frac{\partial f_{\mathrm{F}}(\epsilon)}{\partial \epsilon} \sum_{n} \epsilon_{n} \operatorname{Im}\left\langle G_{n n}\left(\epsilon_{+0}\right)\right\rangle .
$$

Finally, we obtain the Hall conductivity $\sigma_{x y}(T, \mu)=\sigma_{x y}^{I}(T, \mu)+\sigma_{x y}^{I I}(T, \mu)$.

\subsection{Thermoelectric coefficients}

In the following we present the numerical results of the thermoelectric coefficients as a function of the chemical potential.

4.2.1. Resistivities $\rho_{x x}$ and $\rho_{x y}$

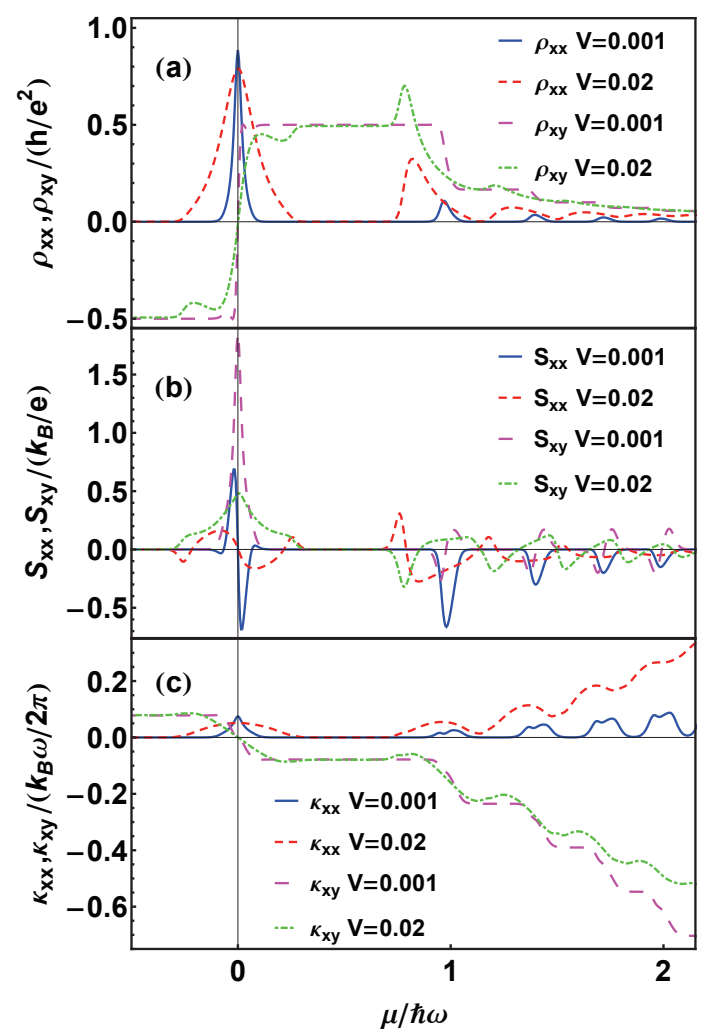

Fig. 1. (Color online) (a) The longitudinal resistivity $\rho_{x x}$ and Hall resistivity $\rho_{x y}$, (b) the Seebeck coefficient $S_{x x}$ and Nernst coefficient $S_{x y}$, (c) the thermal conductivity $\kappa_{x x}$ and thermal Hall conductivity $\kappa_{x y}$ as a function of $\mu$ with different $V$ at temperature $T=5 \mathrm{~K}$.

Using Eqs. (35), (39), and (42) we can obtain the conductivities $\sigma_{x x}$ and $\sigma_{x y}$, numerically. The longitudinal resistivity $\rho_{x x}$ and the Hall resistivity $\rho_{x y}$ are obtained by using the equations, $\rho_{x x}=\sigma_{x x} /\left(\sigma_{x x}^{2}+\sigma_{x y}^{2}\right)$ and $\rho_{x y}=-\sigma_{x y} /\left(\sigma_{x x}^{2}+\sigma_{x y}^{2}\right)$.

The longitudinal resistivity $\rho_{x x}$ and the Hall resistivity $\rho_{x y}$ are depicted in Fig. 1(a) with several values of $V$. Considering that $\rho_{x x}$ is symmetric and $\rho_{x y}$ is antisymmetric with respect to $\mu=0$, only the $n \geq 0$ LL are shown in Fig. 1(a). As it is expected $\rho_{x x}$ displays the peaks at the LLs and vanishes between each consecutive LL, while $\rho_{x y}$ exhibits the typical Hall plateaus. These longitudinal resistivity $\rho_{x x}$ and Hall resistivity $\rho_{x y}$ are closely related to the localization picture in the 2D integer quantum Hall effect(IQHE), i.e., the extended states are at the center of the broaden LLs and all the other states are localized. The fluctuation of impurity scattering 
removes the sharpness of the LLs so that the LLs are broadened as shown in Fig. 1(a). For the zeroth LL $(n=0)$, it is the charge neutrality point of graphene. When the chemical potential is varied through the zeroth LL, a zero-crossing of Hall resistivity $\rho_{x y}$ changes smoothly through zero from its negative quantized value on the hole side to a positive quantized value on the electron side, whereas $\rho_{x x}$ moves from a zero on the hole side through a maximum at the charge neutrality point to another zero on the electron side. Our numerical results of the resistivity agree with the observations in Kim's experiment ${ }^{[16]}$. These results demonstrate that the system is in the Hall regime. The temperature dependence of the $\rho_{x x}$ at the charge neutrality point displays an activated behavior. For the other LLs, the above picture does not change qualitatively. As temperature increases, the peak values of $\rho_{x x}$ decrease and the half widths increase for $n>0$ LLs as shown in Fig. 2(a).

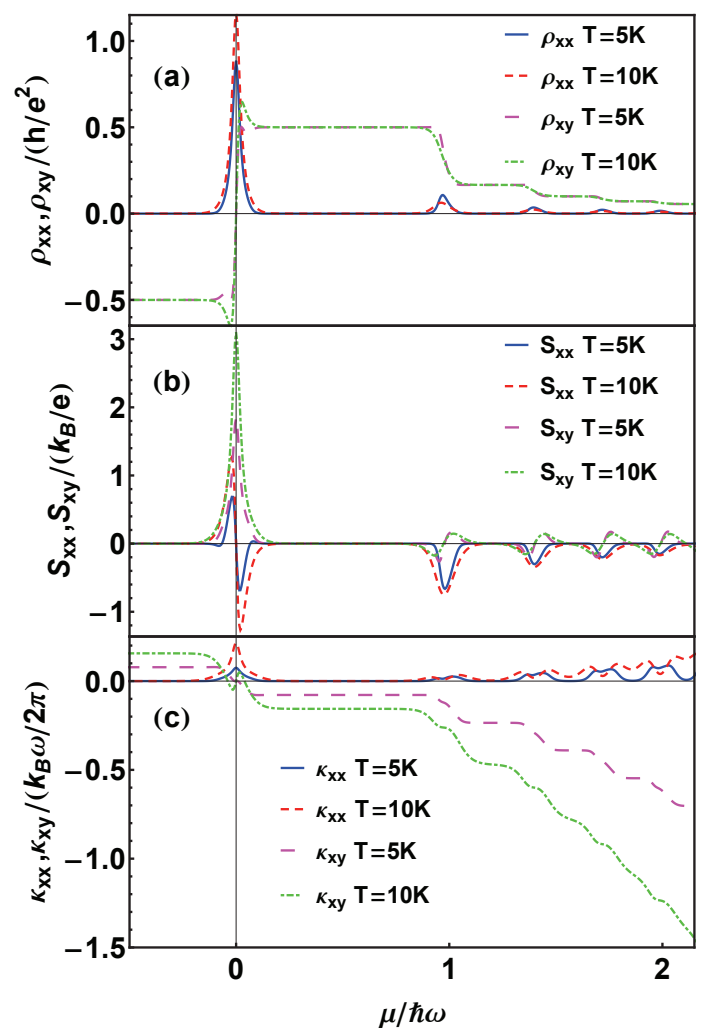

Fig. 2. (Color online) (a) The longitudinal resistivity $\rho_{x x}$ and Hall resistivity $\rho_{x y}$, (b) the Seebeck coefficient $S_{x x}$ and Nernst coefficient $S_{x y}$, (c) the thermal conductivity $\kappa_{x x}$ and thermal Hall conductivity $\kappa_{x y}$ as a function of $\mu$ at different temperatures with $V=0.001$.

\subsubsection{Seebeck coefficient $S_{x x}$ and Nernst coefficient $S_{x y}$}

The Seebeck coefficient $S_{x x}$ and Nernst coefficient $S_{x y}$ are presented in Fig. 1(b) and 2(b). $S_{x x}$ is antisymmetric and $S_{x y}$ is symmetric with respect to $\mu=0$. It is found that as a function of chemical potential, the Seebeck coefficient $S_{x x}$ displays a series of peaks at the LLs for $\mu<0$ and dips at the LLs for $\mu>0$, respectively. $\left|S_{x x}\right|$ has a maximum while the sign of $S_{x y}$ is alternated around the LLs $(n \geq 1)$, corresponding to the extended states at the LLs. The appearance of zero thermoelectric response can be explained by the existence of the localized states in the gap between adjacent LLs, similar to the longitudinal conductivity in the IQHE. $\boldsymbol{S}$ is defined by $\boldsymbol{\rho} \cdot \boldsymbol{\alpha}$, where $\boldsymbol{\alpha}=(1 / T)\left[(\mu / e) \mathbb{L}_{C C}+\mathbb{L}_{C E}\right]$. From this, we have $S_{x x}=\rho_{x x} \alpha_{x x}-\rho_{x y} \alpha_{x y}$ and $S_{x y}=\rho_{x y} \alpha_{y y}+\rho_{x x} \alpha_{x y} \cdot \rho_{a a}$ and $\alpha_{a b}(a \neq b)$ are symmetric functions of $\mu$, while $\rho_{a b}$ and $\alpha_{a a}$ are antisymmetric functions of $\mu$. As the Fermi level passes through the core of extended states where the longitudinal resistance becomes appreciable and the Hall resistance makes its transition from one plateau step to the next. 
Their combination leads the oscillatory structure of $S_{x y}$ in the vicinity of the LLs. As shown in Fig. 1(b), the broadening of LLs due to the impurity scattering increases the width of the peaks or dips for Seebeck coefficient $S_{x x}$ and oscillations of Nernst coefficients $S_{x y}$. As temperature increases, $S_{x x}$ increases and $S_{x y}$ decreases for $n \geq 1$ LLs as shown in Fig. 2(b) .

The Seebeck and Nernst coefficients in the vicinity of the zeroth LL have an opposite behavior compared with those in the higher LLs. Instead of $S_{x x}$, the Nernst coefficient $S_{x y}$ displays a large peak while the Seebeck coefficient $S_{x x}$ now displays an alternate sign. The feature at $n=0$ LL reflects the characteristic property of 2D relativistic fermions in the perpendicular magnetic field. Around the zeroth LL $(n=0)$, the Hall resistivity change the sign because the charges have opposite sign. As analysis above, Seebeck coefficient from the edge states is larger when the chemical potential is below the LL, and the resistivity for the holelike quasiparticles makes $S_{x x}$ negative. When the chemical potential lies near the top of the lowest LL, i.e., in the electron side, the $S_{x x}$ has a similar behavior as that in the higher LLs. Hence, the Seebeck coefficient $S_{x x}$ displays an alternate sign. Similarly, the Hall effect with the carrier flux driven by the temperature gradient predominates the Nernst coefficient when the chemical potential near the edge of band lies below the LL. So $S_{x y}$ is positive. Binding this with that in the electron side leads to occur a maximum of $S_{x y}$ in the vicinity of the lowest LL. Experimentally $S_{x x}$ changes the sign from a dip to a peak with increasing $\mu$ around $\mu=0$. This discrepancy with our results is related to the lower $\rho_{x x}$ value when $\mu$ is at the $n=0 \mathrm{LL}$, which is also observed in other theoretical calculations by different methods ${ }^{[44,45]}$. Apart from this, the Seebeck coefficent $S_{x x}$ and the Nernst coefficient $S_{x y}$ are in good agreement with the experimental results ${ }^{[15-17]}$.

\subsubsection{Thermal conductivities $\kappa_{x x}$ and $\kappa_{x y}$}

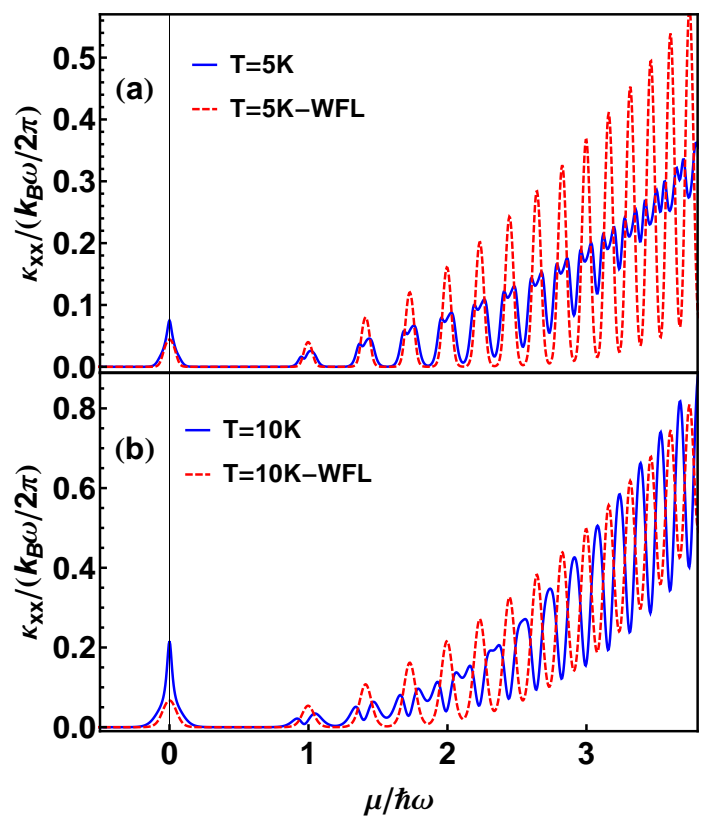

Fig. 3. (Color online) The thermal conductivity $\kappa_{x x}$ as a function of $\mu$ with $V=0.001$ at (a) $T=5 \mathrm{~K}$ and (b) $T=10 \mathrm{~K}$, respectively. The results calculated from the Wiedemann-Franz law by conductivity $\sigma_{x x}$ are labeled by WFL in the legends.

Fig. 1(c) and 2(c) show the thermal conductivities as a function of chemical potential $\mu$ for $n \geq 0 \mathrm{LL}$. The longitudinal component of thermal conductivity $\kappa_{x x}$ is symmetric while the transverse component $\kappa_{x y}$ is antisymmetric with respect to $\mu=0 . \kappa_{x x}$ shows a peak and $\kappa_{x y}$ jumps to a new plateaus when the chemical 
potential equals $\epsilon_{n}=\operatorname{sgn}(n) \hbar \omega \sqrt{|n|}$. These behaviors are similar to those for the conductivities (resistivities). However, the peaks of $\kappa_{x x}$ at those higher LLs split into two peaks which also been shown in Ref. [47] and these split double peaks smear to one peak with increasing the impurity. In addition, the transverse thermal conductivity exhibits a sign reversing behavior when the chemical potential skims over the zeroth LL as shown in Fig. 2(c). The sign reversing in $\kappa_{x y}$ implies the effects of thermally excited electrons and holes for the zeroth LL state. The thermal conductivity contributions arising from electrons and holes are not simply additive, but in combination they give rise to an additional contribution known as the bipolar effect. In order to check the validity of the Wiedemann-Franz law, the thermal conductivity calculated by $\kappa_{x x}=L_{0} \sigma_{x x} T$ where $L_{0}=\pi^{2} k_{\mathrm{B}}^{2} /\left(3 e^{2}\right)$ are shown in Fig. 3 for comparison with the numerical results. We see that at the central region of the LL, the Wiedemann-Franz law is violated. As shown in Fig. 3(b), because of the split double peak structure of $\kappa_{x x}$ and the non-constant space between nearest LLs, the peaks of nearest LL merge into one peak when $n>6$ which is the origin of the out of phase oscillations with electrical conductivity in Ref. [43], eventhough the double peaks are clear when $n<6$. At low temperature, the broaden of the LLs is narrow and the double peak structure is still clear as shown in Fig. 3(a). It is found that the enhance of impurity scattering results in enhancing $\kappa_{x x}$ and suppressing $\kappa_{x y}$ as shown in Fig. 1(c). This double peak structure of longitudinal thermal conductivity in high LLs and the anomalous jump at the zeroth LL with a sign reversal in the transverse thermal conductivity have not yet been observed experimentally.

From the thermal and electrical transport coefficients, the thermoelectric figure of merit $Z T$ is calculated and is shown in Fig. 4. $Z T$ exhibits resonant like structure. The magnetically quantized graphene is a very good thermoelectric materials when the chemical potential is pinned to first LLs. This excellent $Z T$ can be the basis for expanding thermoelectrical application of graphene ${ }^{[66,67]}$. For practical applications with sufficient power output, graphene with high carrier concentration is required. This would require a high magnetic field to locate the chemical potential in the first LL. A trade-off between the efficiency $Z T$ and the power needs to be considered to determine the optimal condition.

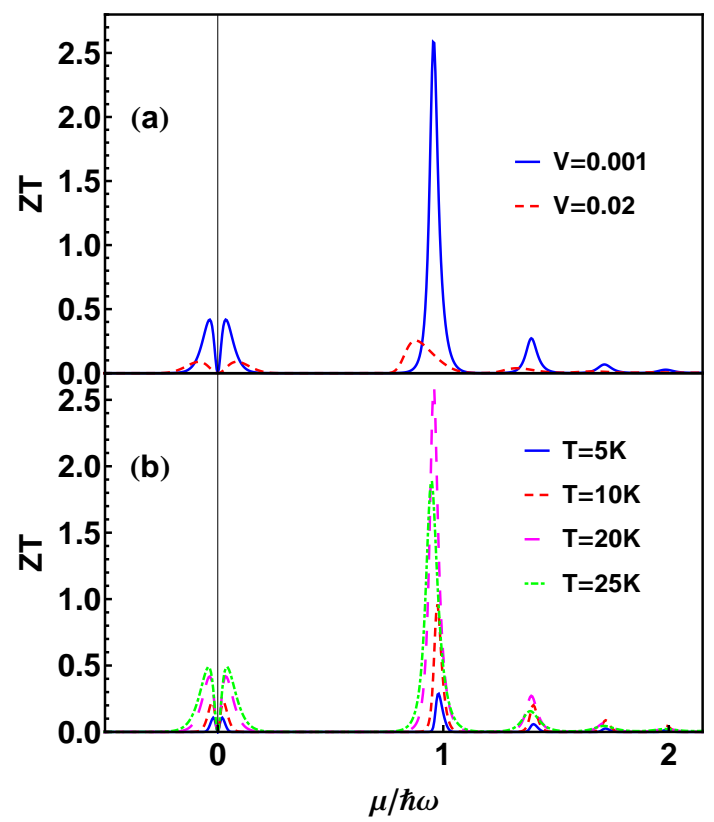

Fig. 4. (Color online) The thermoelectric figure of merit as a function of $\mu$ (a) at $T=20 \mathrm{~K}$ with different $V$ and (b) with $V=0.001$ at different temperatures. 


\section{Summary}

We have calculated the charge and thermal transport coefficients in magnetically quantized graphene in the presence of intra-valley impurity scattering. The Green's function and the self-energy were calculated in SCBA. A detailed investigation of the electrical and thermal properties of graphene in a magnetic field is performed by means of the Kubo-Strěda formula in the presence of impurity scattering and thermal broadening. The results agree with experiments qualitatively. The features of Seebeck coefficient and the transverse thermal conductivity at the zeroth LL are discussed associated with the intensity of impurity scattering. The sign reversal in Seebeck coefficient and the transverse thermal conductivity imply the effects of thermally excited electrons and holes for the zeroth LL state. The impurity scattering may result a bipolar effect from these thermally excited electrons and holes. Finally, our results of the thermoelectric figure of merit suggest that an external strong magnetic field could be used to effectively enhance the $Z T$.

\section{References}

[1] Novoselov K S et al. 2004 Science 306666

[2] Novoselov K S et al. 2005 Nature 438197

[3] Katsnelson M I, Novoselov K S and Geim A K 2006 Nat. Phys. 2620

[4] Neto A H C, Guinea F, Peres N M R, Novoselov K S and Geim A K 2009 Rev. Mod. Phys. 81109

[5] Sarma S D, Adam S, Hwang E H and Rossi E 2011 Rev. Mod. Phys. 83407

[6] Zhang Y, Tan Y W, Stormer H L and Kim P 2005 Nature 438201

[7] Zhang Y et al. 2006 Phys. Rev. Lett. 96136806

[8] Zhang Z, Zhang Y, Stormer H L and Kim P 2007 Phys. Rev. Lett. 99106802

[9] Abanin D A, Novoselov K S, Zeilter U, Lee P A, Geim A K and Levitov L S 2007 Phys. Rev. Lett. 98 196806

[10] Checkelsky J G, Li L and Ong N P 2008 Phys. Rev. Lett. 100206801

[11] Checkelsky J G, Li L and Ong N P 2009 Phys. Rev. B 79115434

[12] Giesbers A J M et al. 2009 Phys. Rev. B. 80 201403(R)

[13] Bolotin K I, Ghahari F, Shulman M D, Stormer H L and Kim P 2009 Nature 462196

[14] Du X, Skachko I, Duerr F, Luican A and Andrei E Y 2009 Nature 462192

[15] Wei P, Bao W, Pu Y, Lau C N and Shi J 2009 Phys. Rev. Lett. 102166808

[16] Zuev Y M, Chang W and Kim P 2009 Phys. Rev. Lett. 102096807

[17] Checkelsky J G and Ong N P 2009 Phys. Rev. B 80 081413(R) 
[18] Liu X, Wang D, Wei P, Zhu L and Shi J 2012 Phys. Rev. B 86155414

[19] Nam Y, Sun J, Lindvall N, Yang S J, Park C R, Park Y W and Yurgens A 2014 Appl. Phys. Lett. 104 021902

[20] Wang D and Shi J 2011 Phys. Rev. B 83113403

[21] Ghahari F, Xie H-Y, Taniguchi T, Watanabe K, Foster M S and Kim P 2016 Phys. Rev. Lett. 116136802

[22] Xu Y, Li Z and Duan W 2014 Small 102182

[23] Sadeghi H, Sangtarash S and Lambert C J 2015 Beilstein J. Nanotechnol. 61176

[24] Zheng H, Liu H J, Tan X J, Lv H Y, Pan L, Shi J and Tang X F 2012 Appl. Phys. Lett. 100093104

[25] Olaya D, Morales M H , Gó mez D, Uribe O A C, Juang Z-Y, and Herná ndez Y 2018 2D Mater. 5011004

[26] Yadav S, Chaudhary S and Pandya D K 2018 Ceram. Int. 4410628

[27] Cheng X and Sun G-Y 2017 Physica E 8784

[28] Nguyen M C, Nguyen V H, Nguyen H-V, Martin J S and Dollfus P 2015 Physica E 73207

[29] Vishkayi S I and Soleimani H R 2015 Physica E 74363

[30] Patil U and Muralidharan B 2017 Physica E 8527

[31] D'Souza R and Mukherjee S 2016 Physica E 8196

[32] Rodriguez S T, Grosu I, Crisan M and Tifrea I 2018 Physica E 961

[33] Valdovinos S M, Rivera J M, Cabrera N E M and Vargas I R 2018 Physica E 101188

[34] Tran V-T, Martin J S, Dollfus P and Volz S 2017 Sci Rep 72313

[35] Anno Y, Imakita Y, Takei K, Akita S and Arie T 2017 2D Mater. 4025019

[36] Zberecki K, Swikowicz R, Wierzbicki M and Barnas J 2016 Phys. Chem. Chem. Phys. 1818246

[37] Kolesnikov D V, Lobanov D A and Osipov V A 2016 Solid State Common. 24883

[38] Kim J Y and Grossman J C 2015 Nano Lett. 152830

[39] Nam S G, Ki D K and Lee H J 2010 Phys. Rev. B 82245416

[40] Wang C R, Lu W S and Lee W L 2010 Phys. Rev. B 82121406

[41] Zhu Z W, Yang H, Fauqu B, Kopelevich Y and Behnia K 2010 Nature Phys. 626

[42] Gusynin V P and Sharapov S G 2005 Phys. Rev. B 71125124

[43] Dóra B and Thalmeier P 2007 Phys. Rev. B 76035402

[44] Zhu L, Ma R, Sheng L, Liu M and Sheng D-N 2010 Phys. Rev. Lett. 104076804 
[45] Chao S -P and Aji V 2011 Phys. Rev. B 84155430

[46] Ugarte V, Aji V and Varma C M 2011 Phys. Rev. B 84165429

[47] Nasir R and Sabeeh K 2011 J. Phys: Condens. Matt 23375301

[48] Proskurin I and Ogata M 2013 J. Phys. Soc. Jpn. 82063712

[49] Xing Y, Sun Q-F and Wang J 2009 Phys. Rev. B 80235411

[50] Wei M-M, Zhang Y-T, Guo A-M, Liu J-J, Xing Y and Sun Q-F 2016 Phys. Rev. B 93245432

[51] Saito K, Nakamura J and Natori A 2007 Phys. Rev. B 76115409

[52] Yiğen S and Champagne A R 2014 Nano Lett. 14289

[53] Crossno J et al. 2016 Science 3511058

[54] Ajiki H and Ando T 1993 J. Phys. Soc. Jpn. 621255

[55] Ajiki H and Ando T 1996 J. Phys. Soc. Jpn. 65505

[56] Ando T and Nakanishi T 1998 J. Phys. Soc. Jpn. 671704

[57] Ando T 2000 Semicond. Sci. Technol. 15 R13

[58] Ando T 2005 J. Phys. Soc. Jpn. 74777

[59] Shon N H and Ando T 1998 J. Phys. Soc. Jpn. 672421

[60] Zheng Y and Ando T 2002 Phys. Rev. B 65245420

[61] Smreka L and Strěda P 1977 J. Phys.: Solide State Phys. C 102153

[62] Luttinger J M 1964 Phys. Rev. 135 A1505

[63] Jonson M and Girvin S M 1984 Phys. Rev. B 291939

[64] Strěda P 1983 J. Phys. C 16 L369

[65] Oji H and Strěda P 1985 Phys. Rev. B 317291

[66] Vining C B, Nat. Mater. 8 (2009) 83.

[67] Tritt T M, Annu. Rev. Mater. Res. 41 (2011) 433. 